\title{
Estrategias para la prevención de errores en la administración de medicamentos intravenosos*
}

\author{
Strategies for the Prevention of Errors in the Administration of Intravenous Medication \\ Estratégias para a prevenção de erros na administração de medicamentos intravenosos
}

\author{
Maria Zoraida Rojas-Marín ${ }^{\text {a }}$ \\ Universidad Nacional de Colombia, Colombia \\ mrojasm@unal.edu.co \\ ORCID: https://orcid.org/0000-0002-1228-9214 \\ Cristian David Castillo-Penagos \\ Universidad Nacional de Colombia, Colombia \\ ORCID: https://orcid.org/0000-0002-1523-1843 \\ Yenifer Daniela Orduz-Díaz \\ Universidad Nacional de Colombia, Colombia \\ ORCID: https://orcid.org/0000-0002-0500-1411 \\ Mayra Alejandra Segura-Suárez
Universidad Nacional de Colombia, Colombia
ORCID: https://orcid.org/0000-0002-3758-712x
}

DOI: https://doi.org/10.11144/Javeriana.ie23.epea

Recibido: 30 Mayo 2021

Aceptado: 30 Agosto 2021

Publicado: 19 Noviembre 2021

\section{Resumen:}

Los medicamentos que son administrados erróneamente pueden producir efectos adversos, los cuales pueden causar afectaciones en el paciente y gastos adicionales en las instituciones. Los cuatro errores más comunes en la administración de medicamentos por vía intravenosa son dosis incorrectas del medicamento, volumen incorrecto, mezclas incorrectas e incompatibilidad farmacológica. El nivel de conocimiento farmacológico en el profesional de enfermería cobra especial importancia. Objetivo: analizar la literatura disponible sobre estrategias utilizadas para prevenir la ocurrencia de estos errores en la administración de medicamentos intravenosos por parte del personal enfermería desde el año 2010 al 2019. Método: se realizó una revisión integrativa (RI) para dar respuesta a la pregunta: ¿cuál es la evidencia disponible sobre estrategias para la prevención de errores en la administración de medicamentos por vía intravenosa? Se utilizaron bases datos entre las que se encuentran OVID, ScienceDirect, SciELO, Medline Complete, Academic Search Complete, PubMed y Guías de Prácticas Clínicas, con límite de tiempo entre 2010 y 2019. Resultado: se identificaron 17 publicaciones sobre estrategias de prevención de errores en la administración de medicamentos por vía Intravenosa, se clasificaron los resultados en 3 grupos relacionados con dosis, volumen, mezclas e incompatibilidades y con factores extrínsecos del paciente. Conclusiones: el número de estudios en cuanto a la prevención de errores en la administración de los medicamentos intravenosos es reducido al igual que el rigor metodológico; respecto a las estrategias de minimización de errores, prevalece la utilización de dispositivos electrónicos (bombas inteligentes, códigos electrónicos, etiquetado), comunicación asertiva, creación y adherencia a las guías y protocolos (principalmente en fármacos analgésicos y antibióticos), fortalecimiento de los conocimientos individuales (educación continua, temas farmacológicos y capacitación en habilidades técnicas) y la generación de una estandarización en la preparación y administración de medicamentos intravenosos.

Palabras clave: administración intravenosa, errores de medicación, seguridad del paciente.

\section{Abstract:}

Erroneous intravenous medication administration can produce adverse effects, which can cause affectations in the patient and additional expenses for the institutions. The four most common errors in intravenous medication administration are wrong doses, wrong volume, wrong mixes, and pharmacological incompatibility. The level of pharmacological knowledge in nursing professionals is essential. Objective: to analyze the available literature on strategies used to prevent these errors in the administration of intravenous medications by nursing staff from 2010 to 2019. Method: an integrative review (IR) was carried out to answer the question: what is the available evidence on strategies for preventing errors in the administration of intravenous medication?

Notas de autor

\footnotetext{
a Autora de correspondencia. Correo electrónico: mrojasm@unal.edu.co
} 
Databases were used, including OVID, ScienceDirect, SciELO, Medline Complete, Academic Search Complete, PubMed, and Clinical Practice Guidelines, with a period limit between 2010 and 2019. Results: 17 publications were identified on strategies for preventing errors in the administration of intravenous medication. The results were classified into 3 groups related to dose, volume, mixtures, and incompatibilities and with factors extrinsic to the patient. Conclusions: the number of studies regarding the prevention of errors is small, as is the methodological rigor. Regarding the strategies to minimize errors, the use of electronic devices prevails (smart pumps, electronic codes, labeling); assertive communication, creation, and adherence to guidelines and protocols (mainly in analgesic drugs and antibiotics); strengthening of individual knowledge (continuing education in pharmacological issues and training in technical skills) and the generation of standardization in the preparation and administration of intravenous medication.

Keywords: intravenous administration, medication errors, patient safety.

\section{Resumo:}

Os medicamentos administrados de forma incorreta podem produzir efeitos adversos, que podem causar afetações no paciente e gastos adicionais nas instituições. Os quatro erros mais comuns na administração intravenosa de medicamentos são doses erradas, volume errado, misturas erradas e incompatibilidade farmacológica. O nível de conhecimento farmacológico do profissional de enfermagem é especialmente importante. Objetivo: analisar a literatura disponível sobre as estratégias utilizadas para prevenir a ocorrência desses erros na administração de medicamentos intravenosos por profissionais de enfermagem no período de 2010 a 2019. Método: foi realizada uma revisão integrativa (RI) para responder à questão: Quais as evidências disponíveis sobre estratégias de prevenção de erros na administração de medicamentos intravenosos? Foram utilizadas bases de dados, entre as que estao OVID, ScienceDirect, SciELO, Medline Complete, Academic Search Complete, PubMed e Guías de Práticas Clínicas, com limite de tempo entre 2010 e 2019. Resultado: foram identificadas 17 publicações sobre estratégias de prevenção de erros na administração de medicamentos por via intravenosa, os resultados foram classificados em três grupos relacionados a dose, volume, misturas e incompatibilidades e a fatores extrínsecos ao paciente. Conclusões: o número de estudos sobre prevenção de erros na administração de medicamentos intravenosos é pequeno, assim como o rigor metodológico. Em relação às estratégias para minimizar erros, prevalece o uso de dispositivos eletrônicos (bombas inteligentes, códigos eletrônicos, rotulagem), comunicação assertiva, criação e adesão a diretrizes e protocolos (principalmente em fármacos analgésicos e antibióticos), fortalecimento dos conhecimentos individuais (educação continuada, questões farmacológicas e treinamento em habilidades técnicas) e a geração de uma padronização no preparo e administração de medicamentos intravenosos.

Palavras-chave: administração intravenosa, erros de medicação, segurança do paciente.

\section{Introducción}

La terapia farmacológica es una de las herramientas más útiles dentro de la práctica de las ciencias de la salud, por lo que su uso se constituye como una de las actividades con gran impacto en la recuperación de los pacientes (1). En esta participan varios profesionales de la salud, por lo que los errores pueden presentarse desde la prescripción, procesamiento de la orden, dispensación del medicamento, preparación, hasta la administración (2). La fase de administración de los medicamentos es una de las intervenciones de enfermería más frecuentes dentro de los diferentes servicios hospitalarios, en donde el Consejo Internacional de Enfermería (CIE) (3) plantea directamente esta labor como una función esencial, así como una responsabilidad permanente del profesional de enfermería, quien\#debe reafirmar sus conocimientos y aptitudes para la administración de un fármaco y, a su vez, evaluar los diferentes factores relacionados, tales como la farmacocinética, farmacodinamia, variables individuales que afectan la acción de los medicamentos, tipos de prescripciones y vías de administración, con el fin de evitar errores relacionados con la administración (4).

Los medicamentos administrados erróneamente pueden producir serios perjuicios y daños al paciente; que se clasifican en categorías que, van desde lesiones en el sistema nervioso central, hipersensibilidad al medicamento, amputación de miembros, alteración de los órganos de los sentidos y dolor, hasta la muerte (5). Estudios han demostrado que la prevalencia de los errores de medicación ocurre en un $67 \%$ la etapa prescripción y un $46 \%$ en la administración (4). En Colombia, en 2017 se registraron 723 reportes 
relacionados a errores de medicación, donde el $15 \%$ del total fueron debido a errores en la administración y en un $14 \%$ de estos se generó un daño al paciente (6).

Para la administración de medicamentos se utilizan diferentes vías tales como oral (VO), intramuscular (IM), subcutánea (SC) e intravenosa (IV), entre las más comunes. La vía intravenosa es una de las más usadas en el ámbito intrahospitalario por su inicio rápido de la acción terapéutica. Sin embargo, es una de las vías con mayor número de reportes de errores de medicación, con un $54 \%$ de reportes en eventos adversos y un $56 \%$ de reportes relacionados con el fármaco (7). Estudios en instituciones hospitalarias han logrado identificar y definir los cuatro errores más comunes en la administración de medicamentos por vía intravenosa: dosis del medicamento incorrecta en un 73,3\%; volumen incorrecto en un 33,3\%; mezclas incorrectas en un 5,8 \%; e incompatibilidad farmacológica en un $0,8 \%$ (8). Adicionalmente, se han reportado otros errores que no son tan frecuentes, pero deben ser mencionados, como el tiempo de infusión inadecuado, la vía y el medicamento incorrectos (8).

Se deben considerar diferentes factores que conllevan a los errores durante la administración de medicamentos intravenosos, tales como los intrínsecos (propios del paciente), extrínsecos (atención en salud) y fisicoquímicos, asociados al fármaco ( $\mathrm{pH}$ y osmolaridad) (9). Dentro de los factores intrínsecos a considerar, se encuentran las características físicas y anatómicas del paciente, se debe conocer la morfología circulatoria para definir el vaso a puncionar a la hora de tomar un acceso venoso, el calibre de vena y la calidad de esta; se debe valorar la permeabilidad del acceso, puesto que, si el paciente tiene alteraciones de la coagulación, puede taponar el catéter y retardar la administración de medicamentos. También se debe considerar la edad del paciente, el género y la raza (9). Estudios han demostrado que los principales factores extrínsecos que producen errores en la administración de medicamentos por vía intravenosa, se relacionan con el cálculo de la dosis, una inapropiada preparación del fármaco, soluciones incorrectas y unidades de medidas inadecuadas en la prescripción. Los factores que predisponen dichos errores son las distracciones, con $46 \%$; aumento de la carga de trabajo, con un $24 \%$; poca disponibilidad de personal, con $14 \%$; e inexperiencia por parte del profesional, con $17 \%$ (10). Finalmente, los factores fisicoquímicos de los medicamentos que se deben tener en cuenta son la osmolaridad y el $\mathrm{pH}$ del fármaco. Aquellos medicamentos cuya osmolaridad sea diferente a la sanguínea (280-295 mOsm / litro) causan dolor y flebitis química. Teniendo en cuenta los factores mencionados, se debe seleccionar el sistema de perfusión intravenoso adecuado. Westbrook et al. (8) demuestran que las infusiones intravenosas realizadas por bolo, es decir administradas directamente en el acceso venoso, tienen mayores tasas de error que las infusiones continuas; además, otro aspecto importante a considerar es la experiencia del personal de enfermería, la evidencia señala una tasa de error superior en el personal con experiencia inferior a los 6 años, en comparación con el personal con experiencia superior a este periodo (8). Como práctica segura para la administración de medicamentos, el Ministerio de Salud y Protección Social, en 2014, sugiere por lo menos el uso de "los 5 correctos" en la administración de medicamentos en cualquiera de las vías (11). Debido a la existencia de múltiples variables que aumentan los errores relacionados con la administración de medicamentos, se hace necesario realizar una guía de estandarización para la administración por perfusión intravenosa.

En consecuencia, el desarrollo de la presente RI brindará elementos, tanto de interés académico como disciplinar, al analizar y compilar las estrategias de mayor utilización y efectividad en la administración segura de medicamentos por vía intravenosa; esta información será una base para la actualización del conocimiento, además de brindar las herramientas para la correcta elaboración de actividades con base en la evidencia, fomentando la seguridad y calidad en la atención en salud.

El estudio tuvo como objetivo general analizar la literatura disponible sobre estrategias utilizadas para prevenir la ocurrencia de errores en la administración de medicamentos intravenosos por enfermería desde el año 2010 a 2019, para identificar estrategias de prevención de errores relacionadas con dosis, volumen, factores extrínsecos (distracciones y carga laboral) y otras estrategias para la administración segura de medicamentos. 


\section{Métodos}

Revisión integrativa, luego de identificar el problema, y para responder a la pregunta planteada, ¿cuáles son las estrategias para la prevención de errores en la administración de medicamentos por vía intravenosa disponibles en la literatura en el periodo 2010-2019?, se consideraron como criterios de inclusión para esta revisión, artículos originales o derivados de investigación, que incluyeran información específica de la práctica de enfermería, relacionada con los errores frecuentes en la administración de los medicamentos intravenosos y las estrategias de control de estos. Se llevó a cabo la búsqueda de artículos, guías de práctica clínica y otra bibliografía, utilizando los términos DeCS, Seguridad del paciente, Administración intravenosa y Errores de medicación, y MESH Paciente safety, Intravenous Administration y Medication Errors. Las bases de datos utilizadas para la búsqueda de la bibliografía fueron PubMed, SciELO, ScienceDirect, Google Académico, Ovid Nursing, Medline, y Academic Search Complete. Se incluyeron documentos publicados entre enero de 2010 y junio de 2019, en idiomas inglés, español y portugués. Luego de la búsqueda, se realizó un esquema de selección que incluye los criterios a evaluar de cada artículo, tomando inicialmente el resumen y la metodología de cada uno para poder hacer selección. Se utilizó la estrategia Prisma para organizar la información analizada, el análisis autónomo por cada autor y luego la consolidación de resultados. De forma inicial, con el uso de las palabras clave, se identificaron 2847 artículos, de estos 1183 fueron descartados por ser referencias duplicadas o no cumplir los criterios de inclusión. Se seleccionaron 39 que cumplían criterios y, finalmente, al realizar la lectura del texto completo se incluyeron 17 artículos, los cuales conformaron la muestra total para el análisis de la presente revisión. Este estudio cumplió con los criterios éticos relacionados con la propiedad intelectual. La estrategia de búsqueda y selección de artículos se detalla en la figura 1.

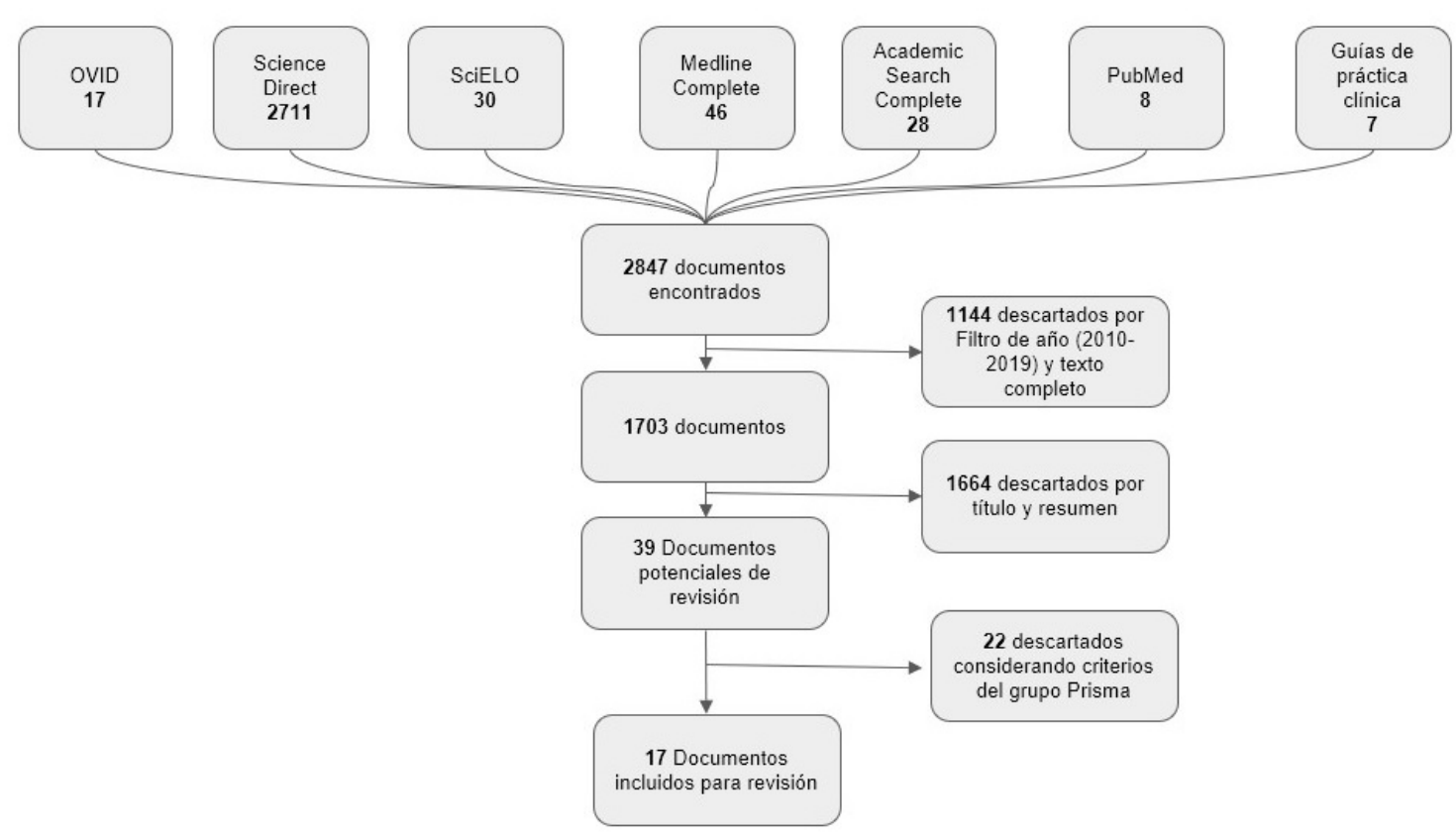

FIGURA 1.

Esquema de búsqueda

Fuente: elaboración propia

\section{Resultados}

En la siguiente tabla se incluyen los 17 documentos con sus respectivos aportes incluidos dentro de la revisión, luego de realizar los diferentes filtros y análisis de pares de autores (ver tabla 1). 
TABLA 1.

Resumen de artículos

\begin{tabular}{|c|c|c|c|}
\hline \multicolumn{4}{|c|}{ Formato de revisión de artículos } \\
\hline & Título & Autores & Principales aportes \\
\hline 1 & $\begin{array}{l}\text { Implementación de estrategias de prevención de } \\
\text { errores en el proceso de administración de } \\
\text { medicamentos: un enfoque para enfermería en } \\
\text { cuidados intensivos }\end{array}$ & Barbagelata (12) & $\begin{array}{l}\text { Énfasis principal en la educación del personal de salud. Los } \\
\text { avances tecnológicos favorecen a la seguridad, pero no se pueden } \\
\text { remplazar por las normas básicas en la seguridad de } \\
\text { administración de medicamentos. }\end{array}$ \\
\hline 2 & $\begin{array}{l}\text { Improving the safety of chcmotherapy } \\
\text { administration: An oncological nurse-led failure } \\
\text { mode and effects analysis }\end{array}$ & Ashley et al. (13) & $\begin{array}{l}\text { Proceso en que al personal de salud se le brindan las herramientas } \\
\text { para disminuir los errores en la administración de quimioterapias } \\
\text { además de reforzar la comunicación entre el personal asistencial y } \\
\text { administrativo. }\end{array}$ \\
\hline 3 & $\begin{array}{l}\text { Intravenous smarts pumps usability issues, } \\
\text { intravenous medication administration error } \\
\text { and patient safety }\end{array}$ & Giuliano (14) & $\begin{array}{l}\text { Los sistemas de perfusión son una herramienta con mucho } \\
\text { potencial para evitar los errores en la administración de } \\
\text { medicamentos por infusiones. Capacitación continua de estas } \\
\text { herramientas. }\end{array}$ \\
\hline 4 & $\begin{array}{l}\text { Medicamentos de administración parenteral: } \\
\text { Recomendaciones de preparación, } \\
\text { administración y estabilidad. }\end{array}$ & Gaspar Carreño et al. (15) & $\begin{array}{l}\text { Establecer una unidad de mezdas parenterales es una forma } \\
\text { efectiva de garantizar eficiencia e idoneidad de la terapia } \\
\text { farmacológica. }\end{array}$ \\
\hline 5 & $\begin{array}{l}\text { Knowledge and practices of nursing concerning } \\
\text { intravenous potassium administration in a } \\
\text { Turkish hospital }\end{array}$ & Güneș, Senol-Celik (16) & $\begin{array}{l}\text { Implementar protocolos y guias de práctica Clínica en cada } \\
\text { hospital, los cuales deben ser aplicables en cada servicio donde las } \\
\text { infusiones de potasio sean frecuentes. }\end{array}$ \\
\hline 6 & $\begin{array}{l}\text { Medication errors in intensive care units: } \\
\text { Nurses' Training needs }\end{array}$ & Simone et al. (17) & $\begin{array}{l}\text { Prácticas sugeridas para mitigar este problema: 1) Talleres } \\
\text { matemáticos para determinar dosis correctas 2) innovación } \\
\text { tecnológica (registro electrónioo de medicamentos, control de } \\
\text { medicamentos de alto riesgo y dosis) 3) Usos de bombas } \\
\text { inteligentes 4) revisión de dosis e infusiones en medicamentos IV } \\
\text { de alto riesgo 5) capacitación constante. }\end{array}$ \\
\hline 7 & $\begin{array}{l}\text { Guía de práctica clínica sobre terapia } \\
\text { intravenosa con dispositivos no permanentes en } \\
\text { adultos }\end{array}$ & $\begin{array}{l}\text { Ministerio de Sanidad, } \\
\text { servicios sociales e igualdad. } \\
\text { España (18) }\end{array}$ & $\begin{array}{l}\text { Su contenido está más dirigido al cuidado del catéter y el } \\
\text { mantenimiento adecuado de la vía endovenosa }\end{array}$ \\
\hline 8 & $\begin{array}{l}\text { Key components of intravenous chemoterapy } \\
\text { labeling: A sistematic review and practice } \\
\text { guideline }\end{array}$ & Trudeau et al. (19) & $\begin{array}{l}\text { Se determinan las recomendaciones para la adecuada elaboración } \\
\text { de las etiquetas cn los fármacos antincoplásicos intravenosos. }\end{array}$ \\
\hline 9 & $\begin{array}{l}\text { A Review of Best Practices for Intravenous } \\
\text { Push Medication Administration }\end{array}$ & Lenz et al. (20) & $\begin{array}{l}\text { Las formulaciones listas para administrar eliminan la necesidad de } \\
\text { compuestos fuera de la farmacia y son proporcionadas por el } \\
\text { fabricante o la farmacia con etiquetado adecuado. }\end{array}$ \\
\hline 10 & $\begin{array}{l}\text { Bombas de infusión continua } \\
\text { de jeringa en unidades neonatales }\end{array}$ & Schmidt et al. (21) & $\begin{array}{l}\text { Deben considerar los factores que alteran el funcionamiento de las } \\
\text { BIC y preferir el uso de jeringas de menor tamaño y flujos } \\
\text { mayores de } 1 \mathrm{~m} / \mathrm{h} \text { para la infusión continua de medicamentos. }\end{array}$ \\
\hline 11 & $\begin{array}{l}\text { Dificultadcs para implementar estrategias de } \\
\text { seguridad del paciente: } \\
\text { Perspectivas de las enfermeras de gestión. }\end{array}$ & Reis et al. (22) & $\begin{array}{l}\text { Se hace necesario contar con servicio de cducación continua y } \\
\text { permanente, sensibilizar e involucrar desde la alta gestión a los } \\
\text { colaboradores de la linea de frente. }\end{array}$ \\
\hline 12 & $\begin{array}{l}\text { Intervenciones de Enfermería para la Seguridad } \\
\text { en la Administración de Medicamentos de Alto } \\
\text { Riesgo en el Adulto }\end{array}$ & Fields, Peterman (23) & $\begin{array}{l}\text { Disponer de protocolos detallados y explíitos, con creación } \\
\text { automática. }\end{array}$ \\
\hline 13 & $\begin{array}{l}\text { Prevención de errores en la administración de } \\
\text { medicamentos en ancstesia }\end{array}$ & Berrío-Valencia (24) & $\begin{array}{l}\text { Importancia sobre la cultura del reporte, al identificar a tiempo los } \\
\text { errores en la administración de medicamentos, se pueden } \\
\text { desarrollar estrategias adecuadas para la prevención. }\end{array}$ \\
\hline 14 & $\begin{array}{l}\text { Procedural and documentation variations in } \\
\text { intravenous infusion administration: a mixed } \\
\text { methods study of policy and practice across } 16 \\
\text { hospital trusts in England }\end{array}$ & Furniss et al. (25) & $\begin{array}{l}\text { Sugiere una estandarización más clara basada en la evidencia y } \\
\text { procedimientos locales que sean contextualmente prácticos para } \\
\text { abordar estos problemas. }\end{array}$ \\
\hline 15 & $\begin{array}{l}\text { Reduction of Medication Errors: a Unique } \\
\text { Approach }\end{array}$ & Schmidt et al. (26) & $\begin{array}{l}\text { Se utiliza una herramienta que involucra la predicción del riesgo } \\
\text { de error en la ejecución de cualquiera de los pasos establecidos } \\
\text { para la administración de medicamentos IV. Donde se puede } \\
\text { estandarizar y racionalizar tres áreas clave de enfoque con el fin de } \\
\text { prevenir errores }\end{array}$ \\
\hline 16 & $\begin{array}{l}\text { Seguridad del paciente en la administración de } \\
\text { antibióticos: evaluación del riesgo. }\end{array}$ & Maqueda-Palau, Pérez (27) & $\begin{array}{l}\text { Se identifican los riesgos con los medicamentos con códigos de } \\
\text { colores y se adopta una matriz de riesgo útil para la evaluación y } \\
\text { detección de los puntos débiles asociados a la preparación y } \\
\text { administración de antibióticos intravenosos }\end{array}$ \\
\hline 17 & $\begin{array}{l}\text { Strategies to minimize medication errors in } \\
\text { emergency units: an integrative review }\end{array}$ & Mieiro et al. (28) & $\begin{array}{l}\text { Se plantearon diferentes estrategias educativas como realización de } \\
\text { campañas, elaboración de manuales explicativos (generación de } \\
\text { unidosis, etiquetas digitales, inclusión de nuevas tecnologís), } \\
\text { creación de una comisión multidisciplinaria involucrada con la } \\
\text { prevención y la reducción de los eventos adversos a los } \\
\text { medicamentos }\end{array}$ \\
\hline
\end{tabular}

Fuente: elaboración propia

Para el análisis de los resultados obtenidos, se identificaron estrategias, las cuales se clasificaron como: estrategias de prevención relacionadas con dosis y volumen, estrategias de prevención relacionadas con mezclas incorrectas e incompatibilidades y estrategias de prevención relacionadas con los factores extrínsecos en la administración de medicamentos por infusión intravenosa.

\section{Estrategias prevención relacionados con dosis y volumen}

Dentro de las recomendaciones halladas en cuanto a dosis y volumen se sugiere:

- Innovación tecnológica con el uso de las bombas de infusión inteligentes $(12,15,23)$.

- Capacitación del personal de enfermería por parte de ingeniería para su correcto manejo y, de esta manera, asegurar el adecuado volumen de perfusión $(12,17,22,23)$.

- El uso de etiquetas a la hora de la preparación del medicamento con información completa (medicamento, unidades, dosis, tipo de diluyente, volumen total a administrar, hora y nombre de quien prepara el medicamento) para demarcar los medicamentos durante su preparación $(12,13$, $18-20,23,24)$. 
- Se sugiere también el uso de unidosis o medicamentos en forma lista para administrar (jeringas prellenadas) $(22,28)$.

- Etiquetas de demarcación electrónicas y/o código de barras $(12,13,18,19)$.

- Compra de medicamentos y soluciones listas para su administración (20,27).

- Se sugiere administrar primero los medicamentos no vesicantes, para así "preparar" la vía para los medicamentos vesicantes (13).

\section{Estrategias de prevención relacionadas con mezclas incorrectas e incompatibilidades fisicoquímicas}

- El uso de guías de práctica clínica, y protocolos de manejo para la estandarización de disoluciones sigue siendo una de las recomendaciones dadas por los diferentes autores (12-14, 16, 17, 25, 26, 28).

- La educación al personal de enfermería en cuanto a los medicamentos y sus diluyentes específicos (17, 27).

- Cada medicamento debe tener de manera explícita en su etiqueta el diluyente a utilizar para evitar incompatibilidades (15).

- No se recomienda el uso de soluciones concentradas, especialmente los electrolitos, los cuales deben estar demarcados con colores llamativos y con etiqueta que sugiere con qué diluir y el volumen para la dilución $(16,19,23)$.

- Si se van a utilizar medicamentos vesicantes, electrolitos o citotóxicos, administrar por una sola vía, y con suficiente disolvente, si el laboratorio fabricante lo recomienda $(18,20)$.

\section{Estrategias de prevención relacionadas con los factores extrínsecos en la administración de medicamentos por infusión intravenosa}

- Se sugiere capacitación al personal de enfermería en cultura de seguridad del paciente y diluciones, en cuanto a equipos asesoramiento, equipos de perfusión y cada una de las interfases que manejen $(12,13,17,21,27)$.

- Se recomienda reducir el número de presentaciones de medicamentos que se dispensan en farmacia (23).

- El entorno de preparación de medicamentos debe ser un área tranquila, en donde el personal cuente con todos los materiales para la preparación y administración del fármaco con el mínimo de distracciones $(13,15)$.

- Por parte del hospital, crear políticas, protocolos y/o guías para la preparación de medicamentos, también la estandarización de las etiquetas que contengan la información anteriormente mencionada $(12-15,17,20,25,28)$.

- Mejorar la comunicación entre el personal y gerencia (13).

\section{Discusión}

La administración de los medicamentos por vía intravenosa es responsabilidad directa del profesional de enfermería (29), pero con influencia de otros profesionales que intervienen en la cadena del medicamento. En la actualidad se reporta un aumento en el número de incidentes derivados de la administración de medicamentos por vía intravenosa, ante lo cual esta revisión permite reflexionar de forma prioritaria en los 
profesionales de enfermería sobre algunas actividades y estrategias que se pueden proyectar en el contexto de la administración segura de medicamentos hacia la mitigación, disminución y eliminación del error (30); y, por consiguiente, reducir las consecuencias negativas para el paciente y las instituciones en relación con los costos (31). Estas estrategias se encuentran igualmente articuladas con la iniciativa de la Organización Mundial de la Salud (OMS), "Medicación sin Riesgo", en el año 2017 (31), con la que se busca reducir a la mitad los errores relacionados con los medicamentos. Esta iniciativa se centra en 4 áreas: pacientes y público, profesionales del área de la salud, medicamentos, sistemas y prácticas de medicación. Respecto al área de los profesionales sanitarios, se busca dar educación y orientación frente al uso correcto de los medicamentos, y así evitar complicaciones, relación coincidente con los resultados de la presente revisión y la de otros autores (32-35). En cuanto al conocimiento práctico de enfermería, en relación con los medicamentos, cobra relevancia debido a la importante necesidad de acciones educativas que fortalecen el conocimiento técnico-científico, con énfasis en cursos y prácticas orientadas a la formación continua en el equipo de enfermería, que generen cada vez más prácticas seguras en relación con la administración de medicamentos, para poder brindar un cuidado óptimo en el que se procure la disminución de eventos adversos relacionados con la medicación. Mientras más conocimiento tenga el profesional de enfermería en cuanto al adecuado uso de los medicamentos, menor será la ocurrencia de errores relacionados con la administración de estos, sobre todo por vía intravenosa (36).

En los resultados de la presente revisión se encontró adicionalmente que los errores más comunes por parte del profesional de enfermería en la administración de medicamentos por vía intravenosa están relacionados con la dosis y volumen, mezclas incorrectas e incompatibilidades y factores extrínsecos en la administración por vía intravenosa, investigaciones con resultados similares (37-40) presentan factores asociados al error, principalmente por desconocimiento farmacológico, fallas en la comunicación, y excesiva carga de actividades y pacientes en las jornadas laborales, que comprometen las intervenciones de cuidado seguro de los profesionales.

A partir del análisis de los artículos, se evidencia que una de las estrategias que más se repiten es el uso de bombas de infusión inteligentes, ya que minimizan los errores que se presentan respecto a la dosis del medicamento-volumen de dilución (36), al tener un mejor control de la dosis a infundir, aunque tienen beneficios mayores, requieren de capacitación adicional al personal (que por lo general presenta alta rotación en los servicios hospitalarios) sobre los diferentes modos de programación y beneficios de uso, programaciones que requieren atención continua para obtener el mayor beneficio, situación que se puede volver contraproducente cuando no se realiza la manipulación de manera adecuada, y de esta manera este recurso tecnológico puede convertirse en un factor que ayude a aumentar este tipo de errores. Por su parte, Arias y García del Barrio (41) encontraron que la utilización de bombas inteligentes ayudó a la disminución de eventos hasta en un $84 \%$, lo que comprueba la efectividad de esta estrategia para contrarrestar los errores relacionados con dosis-volumen. Pero no solo debe generarse capacitación sobre el uso de estos dispositivos, sino también de las características específicas de los medicamentos para sus diluciones, con la construcción de la lista de medicamentos que se instala en estas bombas, que obligue a una revisión de los mismos y de sus protocolos de uso, para evitar prácticas obsoletas y ajustarse a la práctica clínica habitual cuando no se cuenta con farmacopeas (42). También es conveniente precisar que en la programación, con la introducción de las concentraciones de un medicamento, si no se realiza de forma pertinente, también puede favorecer la aparición de errores (43).

Adicionalmente, otro aporte de la presente revisión a considerar en las prácticas seguras de la vía intravenosa por parte de los profesionales de enfermería está encaminado a reconocer el etiquetado como una herramienta muy útil para evitar confusiones al momento de administrar o diluir un medicamento, por ejemplo, el etiquetado electrónico de los medicamentos que incluya información como la dosis a administrar, cuál es el diluyente que se debe utilizar, hora de administración y demás datos que ayudan al profesional de enfermería a reducir equivocaciones; la misma estrategia también es empleada para evitar incompatibilidades medicamentosas frente al diluyente. Para el manejo de las etiquetas en los medicamentos, la OPS, en conjunto 
con su Grupo de Trabajo de Registro de Medicamentos, elaboró un manual de requisitos para el registro de medicamentos en la Américas (44), en el cual sugiere especificaciones para el etiquetado de los medicamentos, que van orientadas hacia el fabricante, las instituciones hospitalarias y el profesional del salud, que tiene a cargo el manejo de medicamentos con especificaciones y demás información que sea útil para evitar errores. El uso de esta estrategia resulta exitoso para prevenir errores relacionados con mezclas incorrectas e incompatibilidades, y también con factores extrínsecos con la comunicación en la administración de medicamentos por vía endovenosa.

El uso de guías de práctica clínica y protocolos de manejo es otra de las estrategias que se debe considerar en el ejercicio del profesional de enfermería, donde autores como González-Expósito (45) y GaravitoAcebes (46) coinciden en que su implementación no solo es útil para prevenir errores relacionados con la administración de medicamentos, sino que además permite a las instituciones la estandarización de este tipo de protocolos. El Ministerio de Salud y Protección Social, en su guía técnica para mejorar la seguridad en la utilización de medicamentos (4), acoge las mejores experiencias de algunas de las instituciones hospitalarias en cuanto a la administración de medicamentos por vía intravenosa, y en todas ellas coinciden en la inclusión de un protocolo de etiquetado de medicamentos y una vigilancia farmacológica, no solo por parte del profesional de enfermería, sino que además se incluya al químico farmacéutico dentro del equipo que participa en la atención del paciente, debido a que aumentaría la efectividad de esta medida. Al estandarizar el proceso de administración de medicamentos, se disminuyeron considerablemente los errores relacionados con dicho proceso (42). A pesar de la evidencia, en Colombia no existen datos referentes a la implementación de guías estandarizadas para la administración de medicamentos. En el estudio de Restrepo (47), se indaga sobre la percepción que tiene el personal de salud sobre las prácticas seguras en la administración de medicamentos, y se encontró que a pesar de que existen protocolos estandarizados a nivel nacional frente a la seguridad del paciente, estos no aseguran la disminución de errores relacionados con la administración de medicamentos, por lo que se requiere la implementación de herramientas de consulta que sean fiables, seguras y actualizadas para la administración de fármacos, que permitan disminuir los errores. Así mismo, este artículo menciona que los aspectos más importantes en estos casos son la administración correcta, disminución de dudas, mejorar la seguridad en el proceso y un doble chequeo (48).

Para cada uno de estos errores se describen estrategias que, de acuerdo con los investigadores, resultan ser exitosas para disminuir el error al que se hace referencia y garantizar la seguridad del paciente durante el proceso de hospitalización. A pesar de todas las actividades y estrategias, puede que no se generen cambios de acuerdo con intervenciones estructurales, sino más bien actividades individuales de profesionales, como lo plantea una revisión de Khalil et al. (49), con el análisis de 30 estudios, donde se determina que no hay suficiente homogeneidad en las investigaciones, y que las intervenciones para reducir los errores de medicación prevenibles hacen pocas o ninguna diferencia en los ingresos y estancias hospitalarias. Sin embargo, desde la postura de los autores de la presente revisión, se debe seguir sensibilizando tanto a los profesionales de enfermería de asistencia directa como a los tomadores de decisiones en las instituciones para seguir fortaleciendo y sumando esfuerzos conjuntos que propicien una mayor cultura de seguridad del paciente.

La identificación de riesgos y fallas es un punto de partida interesante y necesario para la mitigación del error. Pero la operacionalización de las estrategias es una intervención que requiere de un sin número de recursos y, sobre todo, de voluntad tanto individual como institucional, ya que los factores que conllevan al error en la administración son evidentemente variados, como lo refiere Martínez (50), desde la sobrecarga de funciones, complejidad de los pacientes, interrupciones del equipo, hasta distracciones del personal y fallas en los entornos locativos para la administración de medicamentos, sin dejar de mencionar la falta de educación continua y preparación individual en competencias farmacológicas.

El conocimiento producido en las investigaciones analizadas en la presente revisión evidencia una realidad frecuente en los diferentes escenarios de cuidado, donde las intervenciones de enfermería, en la administración 
de medicamentos de forma frecuente y rutinaria en la vía intravenosa, generan una necesidad importante de fortalecer la cultura de seguridad en el uso de medicamentos. El empleo de fármacos es incuestionablemente necesario y depende del conocimiento y preparación del personal profesional, que parten del reconocimiento de las características específicas de la vía intravenosa, las ventajas y desventajas, el análisis clínico, el aprovechamiento de las herramientas y recursos tecnológicos, así como el cumplimiento de los diferentes principios o fundamentos correctos para la administración de la farmacoterapia, y que se genere el objetivo terapéutico con los mayores efectos satisfactorios y la disminución de eventos adversos. La administración de medicamentos por vía intravenosa sigue siendo una responsabilidad casi total de la disciplina de enfermería; los profesionales se deben empoderar y liderar actividades, intervenciones y estrategias de generación de conocimiento en esta área tan importante. Sin embargo, no se puede olvidar que el resultado final se ve integrado con otros actores en la cadena del medicamento, como el personal médico y de farmacia. Generar alianzas con otras disciplinas posibilita una mayor comprensión y actuación integral del proceso, facilitando los hallazgos respecto a los factores causales y consecuentes propuestas de intervención (51).

\section{Limitaciones de la revisión}

Como limitaciones de esta RI, se encuentra la evidencia en la calidad de los estudios analizados, en cuanto al rigor metodológico que describan las intervenciones con mayor detalle, así como la baja producción científica, especialmente la colombiana, sobre el tema, lo que demuestra que se requiere una mayor fundamentación para la administración segura de medicamentos intravenosos.

\section{Conclusiones}

Los errores en la administración de medicamentos intravenosos son una realidad de alta frecuencia, sin embargo, como se presenta en la RI, el número de estudios y publicaciones en cuanto a las estrategias de minimización para estos son limitados $(52,53)$, y más aún a nivel nacional, con evidencias de mediano y bajo rigor metodológico.

Las estrategias que se evidenciaron en la RI para prevención de errores en la administración de medicamentos intravenosos se centran en la utilización de dispositivos electrónicos (bombas inteligentes, códigos electrónicos, etiquetado), comunicación asertiva, creación y adherencia a las guías y protocolos (principalmente en fármacos analgésicos y antibióticos), fortalecimiento de los conocimientos individuales (educación continua, temas farmacológicos y capacitación en habilidades técnicas) y generar una estandarización en la preparación y administración de medicamentos intravenosos.

Sin embargo, como se analiza en esta revisión, son más esfuerzos individuales de profesionales o grupos de instituciones hospitalarias, sin que se presenten recomendaciones estandarizadas o en consenso, y no permiten evidenciar impacto de las estrategias a mediano y largo plazo.

\section{Referencias}

1. Subsecretaría de Salud Pública. División de políticas públicas saludables y promoción Uso racional de medicamentos: una tarea de todos. Santiago de Chile: Departamento Políticas Farmacéuticas y profesiones Médicas; 2010.

2. Marín-Morales A, Rojas-Marín Z, Guarnizo-Tole M, Bonilla-Manchola A. Manual para la administración de medicamentos desde el proceso de atención de enfermería. Bogotá: Universidad El Bosque; 2017.27 p.

3. Domínguez-Martínez M, Pérez-Castro A, Vásquez J, Soto-Arreola M. Eficacia de la práctica de enfermería en la administración de medicamentos. Conamed [Internet]. 2015;20(1):s35-s40. Disponible en: https://www.med igraphic.com/pdfs/conamed/con-2015/cons151f.pdf 
4. Ministerio de Salud y Protección Social. Salud. Mejorar la seguridad en la utilización de medicamentos [Internet]. 2011. Disponible en: https://www.minsalud.gov.co/sites/rid/Lists/BibliotecaDigital/RIDE/DE/CA/segurida d-en-la-utilizacion-de-medicamentos.pdf

5. Tosta de Carvalho V, de Bartolí-Cassiani SE. Errores en la administración de medicamentos: análisis de situaciones relatadas por los profesionales de enfermería. Invest. Educ. Enferm [Internet]. 2001;19(2). Disponible en: http s://revistas.udea.edu.co/index.php/iee/article/view/16808

6. Invima. Programa Nacional de Farmacovigilancia [Internet]. 2016. Disponible en: https://www.invima.gov.co/doc uments/20143/462902/Farmaseguridad-Vol-5-N-6.pdf/d45bec82-ee9f-7284-84b7-a2bb887c0a83

7. Madrigal-Cadavid J, Amariles P. Incompatibilidad de medicamentos intravenosos: revisión estructurada. Ces Medicina [Internet]. 2017;31(1):58-69. Disponible en: http://doi.org/10.21615/cesmedicina.31.1.6

8. Westbrook J, Rob M, Woods A, Parry D. Errors in the administration of intravenous medications in hospital and the role of correct procedures and nurse experience. BMJ Quality \& Safety [Internet]. 2011;20(12):1027-1034. http://doi.org/10.1136/bmjqs-2011-000089

9. Grupo CTO. Manual Farmacología. 8a ed. Ciudad de México: CTO Editorial; 2013.

10. Saucedo-Becerra A, Serrano-Flores FE, Flores-Arcos V, Morales-Olarte E, Santos-García A. Errores frecuentes en la administración de medicamentos intravenosos en pediatría. Rev Enferm IMSS [Internet]. 2008;16(1):19-26 Disponible en: https://www.medigraphic.com/cgi-bin/new/resumen.cgi?IDARTICULO=18921

11. Ministerio de Salud y Protección Social. Resolución Numero 2003 de 2014 [Internet]. 2014. Disponible en: http s://www.minsalud.gov.co/Normatividad_Nuevo/Resoluci\%C3\%B3n\%202003\%20de\%202014.pdf

12. Barbagelata E. Implementación de estrategias de prevención de errores en el proceso de administración de medicamentos: un enfoque para enfermería en cuidados intensivos. Rev Méd Clín Las Condes [Internet]. 2016;27(5):594-604. Disponible en: https://doi.org/10.1016/j.rmclc.2016.09.005

13. Ashley L, Dexter R, Marshall F, McKenzie B, Ryan M, Armitage G. Improving the Safety of Chemotherapy Administration: An Oncology Nurse-Led Failure Mode and Effects Analysis. Onc Nursing Forum [Internet]. 2011;38(6):e436-e444. Disponible en: https://doi.org/10.1188/11.ONF.E436-E444

14. Giuliano K. Intravenous Smart Pumps. Crit Care Nurs Clin North Am [Internet]. 2018;30(2):215-224. Disponible en: https://doi.org/10.1016/j.cnc.2018.02.004

15. Gaspar-Carreño M, Torrico-Martín F, Novajarque-Sala L, Batista-Cruz M, Ribeiro-Goncalves P, Porta-Oltra B, et al. Medicamentos de Administración Parenteral: Recomendaciones de preparación, administración y estabilidad. Farm Hospitalaria [Internet]. 2014;38(6):461-467. Disponible en: https://doi.org/10.7399/fh.2014.38.6.7524

16. Güneş A, Senol-Celik S. Knowledge and practices of nurses concerning intravenous potassium administration in a Turkish hospital. Aust J Adv Nurs [Internet]. 2014;32(1):16-22. Disponible en: https://citeseerx.ist.psu.edu/v iewdoc/download?doi=10.1.1.658.5942\&rep=rep1\&type=pdf

17. Simone E, Tartaglini D, Fiorini S, Petriglieri S, Plocco C, Muzio M. Medication errors in intensive care units: nurses' training needs. Emerg Nurse [Internet]. 2016 [citado 2021 mzo. 8];24(4):24-29. Disponible en: http://doi.or $\mathrm{g} / 10.7748 /$ en.2016.11577

18. Ministerio de Sanidad. Guía de Práctica Clínica sobre Terapia Intravenosa con Dispositivos no Permanentes en Adultos [Internet]. 2014. Disponible en: https://portal.guiasalud.es/wp-content/uploads/2018/12/GPC_541 _Terapia_intravenosa_AETSA_compl.pdf

19. Trudeau M, Green E, Cosby R, Charbonneau F, Easty T, Ko Y, et al. Key components of intravenous chemotherapy labeling: a systematic review and practice guideline. J Oncol Pharm Pract [Internet]. 2011 dic.;17(4):409-424. Disponible en: https://doi.org/10.1177/1078155210385160

20. Lenz JR, Degnan DD, Hertig JB, Stevenson JG. A Review of Best Practices for Intravenous Push Medication Administration. J Infus Nurs. 2017;40(6):354-358. Disponible en: https://doi.org/10.1097/NAN.000000000 0000247

21. Schmidt N, Sáez C, Maturana A. Bombas de infusión continua de jeringa en unidades neonatales. Rev. Chil. Pediatr [Internet]. 2011;82(2):123-128. Disponible en: http://doi.org/10.4067/S0370-41062011000200006 
22. Reis G, Oliveira J, Ferreira A, Vituri D, Marcon S, Matsuda L. Dificultades para implantar estrategias de seguranza do paciente: perspectivas de enfermeiros gestores. Rev Gaúcha Enfermagem [Internet]. 2019;40(Espe). Disponible en: https://doi.org/10.1590/1983-1447.2019.20180366

23. Fields M, Peterman J. Intervenciones de Enfermería para la Seguridad en la Administración de Medicamentos de Alto Riesgo. Nurs Admin Q. 2010;29(1):78-87. https://doi.org/10.1097/00006216-200501000-00013

24. Berrío-Valencia M. Prevención de errores en la administración de medicamentos en anestesia. Revista CES [Internet]. 2014;28(2):307-312. Disponible en https://doi.org/10.21615/ces\%20med.v28i2.2925

25. Furniss D, Lyons I, Franklin B, Mayer A, Chumbley G, Wei L, et al. Procedural and documentation variations in intravenous infusion administration: a mixed methods study of policy and practice across 16 hospital trusts in England. BMC Health Services Res [Internet]. 2018;18(1). Disponible en: https://doi.org/10.1186/s12913-0 18-3025-x

26. Schmidt K, Taylor A, Pearson A. Reduction of Medication Errors: A Unique Approach. J Nurs Care Qual [Internet]. 2017;32(2):150-156. Disponible en: https://doi.org/10.1097/NCQ.0000000000000217

27. Maqueda-Palau M, Pérez JE. Seguridad del paciente en la administración de antibióticos: evaluación del riesgo. Rev Calid Asisten [Internet]. 2017;32(3):178-186. Disponible en: http://doi.org/10.1016/j.cali.2016.10.004

28. Mieiro DB, Oliveira ÉBC, Fonseca REPD, Mininel VA, Zem-Mascarenhas SH, Machado RC. Strategies to minimize medication errors in emergency units: an integrative review. Rev Bras Enferm [Internet]. 2019 feb.;72(Supl. 1):307-314. Disponible en: https://doi.org/10.1590/0034-7167-2017-0658

29. Concha L, Silva G. Conocimiento de administración de medicamentos por vía endovenosa de los estudiantes de sexto, séptimo y octavo semestre de enfermería en una universidad del nororiente de Colombia [Tesis pregrado; internet]. [Medellín]: Universidad CES; 2018. Disponible en: https://repository.ces.edu.co/bitstream/10946/ 4205/1/Conocimiento\%20Administraci\%C3\%B3n\%20Medicamento\%20V\%C3\%ADa\%20Endovenosa.pdf

30. Organización Mundial de la Salud. Promoting rational use of medicines [Internet]. Disponible en: https://www .who.int/medicines/areas/rational_use/en/

31. Organización Mundial de la Salud. Iniciativa mundial para reducir a la mitad los errores relacionados con la medicación en cinco años [Internet]. 2017. Disponible en: https://www.who.int/es/news-room/detail/29-032017-who-launches-global-effort-to-halve-medication-related-errors-in-5-years

32. Montero-Vizcaíno Y, Izquierdo-Santa Cruz M, Vizcaíno-Alonso MC, Montero-Vizcaino YY. Knowledge about practice management of drugs in Nursing. Neonatal resuscitation service. Gynecological and Obstetric Hospital of Guanabacoa. Rev Haban Cienc Méd [Internet]. 2017;16(5):832-838. Disponible en: http://scielo.sld.cu/sci elo.php?script $=$ sci_arttext\&pid $=$ S1729-519X2017000500014\&lng=es

33. Carmona-Torres JM, Baena-Serrano AM, Berral-Jiménez AC, Sotelo-Baena Q, Recio-Andrade B, Recio-Andrade I. Análisis del conocimiento sobre la administración de fármacos. Eur. J. Investig. Health Psychol. Educ. 2015;5(2):233-241. Disponible en: https://doi.org/10.30552/ejihpe.v5i2.115

34. Valderrama-Sanabria ML. Intervención de enfermería en la administración de medicamentos en pediatría. Rev. Cienc. Cuidad. 2018;15(1):72-81. Disponible en: https://doi.org/10.22463/17949831.1224

35. Balseiro-Almario L, Osuna E, Javier-Cabrera D. La prescripción de los medicamentos por los licenciados en enfermería: implicaciones de responsabilidad legal. Rev Conamed [Internet]. 2017;22(3):145-151. Disponible en: https://dialnet.unirioja.es/servlet/articulo?codigo $=6434803$

36. Arias P, García del Barrio M. Bombas de infusión inteligentes para administración de citostáticos. Panorama Actual Med [Internet]. 2014;38(375):399-704. Disponible en: https://gruposdetrabajo.sefh.es/gps/images/stories/pu blicaciones/PAM_375-Julio-699-704.pdf

37. Manrique-Rodríguez S, Sánchez-Galindo A, Fernández-Llamazares C, Calvo-Calvo M, Carrillo-Álvarez Á, Sanjurjo-Sáez M. Administración segura de medicamentos intravenosos en pediatría: 5 años de experiencia de una Unidad de Cuidados Intensivos Pediátricos con bombas de infusión inteligentes. Med Intensiva [Internet]. 2016;40(7):411-421. Disponible en: https://doi.org/10.1016/j.medin.2016.01.011 
38. Valle MMF, Cruz EDA, Santos T, Valle MMF, Cruz EDA, Santos T. Incidentes com medicamentos em unidade de urgência e emergência: análise documental. Rev da Esc Enferm da USP [Internet]. 2017;51:1-6. Disponible en: https://doi.org/10.1590/s1980-220x2016033303271

39. Pérez-Díez C, Real-Campaña JM, Noya-Castro MC, Andrés-Paricio F, Abad-Sazatornil M, Povar-Marco JB. Errores de medicación en un servicio de urgencias hospitalario: estudio de situación para mejorar la seguridad de los pacientes. Emergencias [Internet]. 2017;29(6):412-415. Disponible en: https://medes.com/publication $/ 125891$

40. Thomas L, Donohue-Porter P, Stein-Fishbein J. Impact of Interruptions, distractions, and Cognitive Load on Procedure Failures and Medication Administration Errors. J. Nurs Care Qual. [Internet]. 2017;32(4):309-317. https://doi.org/10.1097/NCQ.0000000000000256

41. Arias P, García del Barrio M. Bombas de infusión inteligentes para administración de citostáticos. Panorama Actual Med [Internet]. 2014;38(375):399-704. Disponible en: https://gruposdetrabajo.sefh.es/gps/images/stories/pu blicaciones/PAM_375-Julio-699-704.pdf

42. Vásquez A, Escobar JI. Apreciaciones de trabajadores de la salud sobre la implementación del sistema de seguridad en la utilización de medicamentos. Univ y Salud [Internet]. 2017;19(1):7-16. Disponible en: http://doi.org/1 $0.22267 /$ rus. 171901.64

43. Barrera E, Domínguez L, Novoa-Carvajal L, Rincón D, Roa L. Protocolo de seguridad del paciente para la administración de medicamentos en el centro de simulación de la universidad cooperativa de Colombia sede Bucaramanga [Tesis de pregrado; internet]. 2015. Disponible en: http://hdl.handle.net/20.500.12494/239

44. Organización Panamericana de la Salud. Requisitos para el registro de medicamentos en las Américas [Internet]. 2013. Disponible en: https://www.paho.org/hq/dmdocuments/2013/Red-PARF-10-2013.pdf

45. González-Expósito J, Martínez-Rioja R, Prado-Amores M, García-Román J, Bustamante-Revuelta E, Pini F. Importancia y grado de conocimiento percibido por las enfermeras de Cantabria sobre farmacología, reacciones adversas medicamentosas e interacciones farmacológicas. Metas de Enfermería [Internet]. 2018;8(21). https:// doi.org/10.35667/MetasEnf.2019.21.1003081294

46. Garavito-Acebes P. Enfermería y Reacciones Adversas a Medicamentos: un rol emergente [Tesis pregrado; internet]. [Madrid]: Universidad Autónoma de Madrid; 2018. Disponible en: https://repositorio.uam.es/handle/10486 1684859

47. Restrepo A. Análisis de la implementación de la política de seguridad del paciente en la utilización de los medicamentos en dos instituciones de nivel I y II del departamento de Risaralda [Tesis pregrado; internet]. [Bogotá]: Universidad Libre de Colombia; 2014. https://repository.unilibre.edu.co/bitstream/handle/10901/ 16053/ANALISIS\%20DE\%20LA\%20IMPLEMENTACION\%20.pdf?sequence=1

48. Pardo-Arias L, León-Reyes M. Administración segura de medicamentos de uso endovenoso en la unidad de cuidados intensivos [Tesis pregrado; internet]. [Bucaramanga]: Universidad de Santander; 2018. Disponible en: https://repositorio.udes.edu.co/handle/001/224

49. Khalil H, Bell B, Chambers H, Sheikh A, Avery AJ. Professional, structural and organisational interventions in primary care for reducing medication errors. Cochrane Database of Systematic Reviews [Internet]. 2017;10:CD003942. https://doi.org/10.1002/14651858.CD003942

50. Martínez A. Errores, factores influyentes y estrategias de prevención frente a la administración de medicamentos [Internet]. [Bogotá]: Universidad de Ciencias Aplicadas y Ambientales; 2018. https://repository.udca.edu.co/bitstream/handle/11158/923/FINAL\%20Correcciones\%20Monografi a\%20Angelica\%20Martinez.\%20\%20FINAL.pdf;jsessionid=AF8552E9B4C119AC523E27CEC45D0544?se quence $=1$

51. Toffoletto MC, Canales MA, Moreira-Arce D, Ordenes-Guerra A, Vergara-Rodríguez CA. Errores en la preparación y administración de medicamentos: una revisión integradora de la Literatura Latinoamericana. Enferm. Glob [Internet]. 2015;14(37):350-360. Disponible en: http://scielo.isciii.es/scielo.php?script=sci_art text\&pid=S1695-61412015000100016\&lng=es 
52. Ramos R, Silva ME, Lopes de Sousa ÁF, Amorim-Batista OM, Batista-Moura ME, Ribeiro AM, et al. Indicadores de calidad en la terapia intravenosa. Rev Cubana Enfermer [Internet]. 2018 jun.;34(2):e1589. Disponible en: h ttp://scielo.sld.cu/scielo.php?script=sci_arttext\&pid=S0864-03192018000200007\&lng=es

53. Camero-Solórzano Y, Meléndez-Mogollón I, Guzmán-Túquerrez S, González Q. Técnica de administración de medicación por vía intravenosa en adultos mayores hospitalizados. Rev Conecta Libertad. 2017;1(3):17-30. Disponible en http://revistaitsl.itslibertad.edu.ec/index.php/ITSL/article/view/61

\section{Notas}

* Artículo original de investigación

Licencia Creative Commons CC BY 4.0

Cómo citar este artículo: Rojas-Marín MZ, Castillo-Penagos CD, Orduz-Díaz YD, Segura-Suárez MA. Estrategias para la prevención de errores en la administración de medicamentos intravenosos. Investig Enferm Imagen Desarr. 2021;23. https://doi.org/10.11144/Javeriana.ie23.epea 